\title{
The push to screen newborns for rare autism-linked genetic conditions
}

\author{
BY LAURA DATTARO
}

28 JULY 2021

\section{Listen to this story:}

https://www.spectrumnews.org/wp-content/uploads/2021/07/audio-ac43a73a-2855-4a6ca07a-483ca3105767-encodings.mp3

Earlier this month, researchers reported unpublished preliminary findings from a pilot drug trial for ADNP syndrome, a rare genetic condition often accompanied by autism.

During the nearly year-long trial, 10 children with the condition, aged 6 to 12 , took a low dose of ketamine. The drug is approved to treat depression and, at higher doses, is used as a sedative. But studies suggest it can also increase expression of the gene ADNP, one copy of which has lost some or all of its function in these children, disrupting their early neurodevelopment.

The results of the trial, led by Alexander Kolevzon, professor of psychiatry and pediatrics at the Icahn School of Medicine at Mount Sinai in New York City, were promising, participants' families were told: The children showed improvements in social behavior, attention, sensory sensitivities and other areas, and they suffered no serious side effects.

Ketamine may not prove helpful for these children longer term, or among larger cohorts in future trials, researchers say. But for some, the results represent a mission-critical step toward screening all newborns for ADNP syndrome, says Sandra Sermone, founder of ADNP Kids Research Foundation, which funded the trial. Without any kind of treatment to change the condition's course, mandatory screening is moot. But "the moment we have a drug that shows efficacy," says Sermone, the parent of a teenager with ADNP syndrome, "we blast for newborn screening."

Sermone isn't the only rare-condition advocate eyeing newborn screening. As the cost of genetic testing drops and treatments inch closer to the clinic, parents and researchers alike are talking more urgently about the need to identify autism-linked genetic conditions - including Angelman, 


\section{Spectrum | Autism Research News}

https://www.spectrumnews.org

Rett, fragile X and DDX3X syndromes — in newborns.

For now, the process to add a condition to the newborn screening list in the United States is lengthy and complicated. A foundation that funds research on Angelman syndrome is supporting the Newborn Screening Reauthorization Act, introduced in January, which would increase the budget for the U.S. newborn screening program from $\$ 11.9$ million to $\$ 31$ million and study ways to accelerate the process, among other things.

In the meantime, individual states are piloting their own expansion programs. A North Carolina optional screening project is evaluating hundreds of conditions - some autism-related — to add to its list in 2022. And New York has created its own add-on, called ScreenPlus, that checks for more than a dozen conditions, including Sanfilippo syndrome, a progressive neurological condition often accompanied by autism.

Despite the benefits - among them a chance to start treatments for some potentially lifethreatening traits in infancy - the prospect of expanded screening raises a host of questions. With so much still unknown about how genes contribute to neurodevelopment, identifying a genetic condition in a newborn can create unnecessary uncertainty around the child's progress. And some traits, including those that simply reflect the range of human neurodiversity, may not warrant any intervention.

"These are the kinds of issues we're all grappling with and discussing," says Huda Zoghbi, professor of molecular and human genetics at Baylor College of Medicine in Houston, Texas. Acknowledging tradeoffs between the need to identify children who can benefit and the concern with finding mutations that may never have effects, she adds, "There's a lot of pieces to this."

Illustration by Nan Cao

\section{Treatment horizon:}

For a condition to merit screening, there must be a reliable way to test for it and strong evidence that an existing treatment, administered shortly after birth, can improve the child's outcome - a policy sometimes referred to as "screen only if you can intervene."

That bar may soon be met for multiple autism-linked genetic conditions, researchers say.

In March, Zoghbi and her colleagues showed that intensive training can delay the onset of motor skills problems and memory loss in a mouse model of Rett syndrome, an autism-related genetic condition that primarily affects girls. The training benefits the mice only if it's done before such 


\section{Spectrum | Autism Research News}

https://www.spectrumnews.org

challenges appear - a time window that would be missed in people without newborn screening.

A small number of girls with the Rett mutation never develop the condition's traits, but behavioral treatments are so safe that there is no reason not to pursue them for anyone who screens positive as a newborn just in case, Zogbhi says. "If you know you have an opportunity to do something about it that could then change the course for thousands of girls, then you do it."

Another team has shown that behavioral training can ease worsening motor skills in a mouse model of DDX3X syndrome. And growth hormone therapy can be "somewhat of a game changer" for people with the autism-linked condition Prader-Willi syndrome, particularly when given as early as 3 to 6 months of age, says Stormy Chamberlain, associate professor of genetics and genome sciences at the University of Connecticut.

Gene therapies come with greater risk and cost than other treatments but are also looking increasingly viable for some autism-linked conditions. Work in Angelman mice has shown that altering gene expression early in development can ease anxiety, repetitive behaviors and epilepsy. One clinical trial of a genetic therapy for Angelman syndrome was put on hold in late 2020, but the drug's promise - as well as that of others in trial, along with one set to go to trial later this year - makes the possibility of newborn screening for Angelman more urgent, Chamberlain says.

"The road to get things onto the newborn screening panel is a steep climb," she says. "In Angelman syndrome, because of the therapeutics, there's a push to do that."

At the moment, adding a new condition to the U.S. list of recommended screenings can take years, says Don Bailey, who served for six years on the federal advisory committee that reviews the evidence for new additions.

Part of the challenge is collecting enough data on rare conditions, he says: To identify even 20 babies for a trial like Kolevzon's might require screening millions of children. And gathering sufficient data may take multiple trials. For now, each advocacy group typically needs to acquire its own condition-specific funding, meaning researchers are starting from scratch each time to fund and set up trials.

In 2016, Bailey launched a pilot infrastructure program called Early Check, which he hopes will serve as a prototype for data collection across multiple rare conditions. The program piggybacks on the blood samples routinely collected at birth in Bailey's home state of North Carolina, where he works as a distinguished fellow in early childhood development at the nonprofit research institute RTI International. Between 500 and 600 families sign up each month to have their newborn screened for a short and evolving list of rare conditions, including fragile $X$ syndrome, Bailey says.

Since the program's launch in late 2018, it has identified four infants with fragile X among 16,000 


\section{Spectrum | Autism Research News}

https://www.spectrumnews.org

tests, which cover about 5 or 6 percent of all babies born in the state annually. It's considerable effort to find those babies, Bailey says, but "we're providing intensive early intervention services for those children."

\section{Ethical questions:}

Early screening for rare conditions stands to benefit children and their families, as well as researchers, scientists say.

Children with autism-linked genetic conditions can have other medical problems, such as heart defects. Newborn screening would ensure that these issues are flagged early, says Silvia De Rubeis, assistant professor of psychiatry at the Seaver Center for Autism Research and Treatment at the Icahn School of Medicine at Mount Sinai in New York City and lead investigator on the DDX3X mouse study.

"Knowledge is always power, right?" De Rubeis says. "It's power for patients — for the young ones and adults - for their families, their advocates and also for us as researchers."

Parents who know about their child's genetic condition can also enroll them in clinical trials, if they exist, and connect with advocacy groups or other support systems. That expands the population available to study the gene involved and might also help to alleviate the catch-22 inherent in gaining approval for newborn screening panels: Adding a condition to the list requires evidence that early treatment works, but early treatments can't be trialed without identifying participants at or near birth.

And newborn screening might also reduce existing disparities in diagnoses that contribute to unequal access to treatment based on race, gender, income or other demographic factors. "You try to think of another program that every baby, every family has free access to," Bailey says. "It's a great public health model and has tremendous equity."

isayildiz / Getty Images

Those benefits to screening need to be carefully weighed against other considerations, experts say, including the inevitable risk of uncovering genetic information some people may prefer not to know.

Many mutations lead to a swath of possible outcomes that aren't apparent until later on. The federal newborn screening committee tries to avoid this sort of "patients in waiting" situation, Bailey says. Some mutations may never affect a child's health at all. Many parents find the 


\section{Spectrum | Autism Research News}

https://www.spectrumnews.org

additional knowledge useful, Bailey says, but it can also create stress and potentially change how parents interact with their child. "The price you pay is watching and waiting," Bailey says.

For some, that price still seems high for rare conditions. ADNP syndrome, for example, accounts for less than 1 percent of people with autism, who in turn make up about only 1 to 2 percent of the population. "Are you going to newborn screen every single kid? I mean, I don't know," Kolevzon says, deliberating out loud.

It could make sense if ketamine or another drug is shown to improve outcomes, he adds. "Would you want to do that from birth? Yeah, I guess you would, assuming it's safe."

Cite this article: https://doi.org/10.53053/PWAX5187 